Revue européenne des sciences sociales

European Journal of Social Sciences

XLIII-131 | 2005

La cumulativité des savoirs en sciences sociales

\title{
Mnémosyne érudite - la théorie comme mémoire
}

Henri Volken

\section{OpenEdition}

\section{Journals}

Édition électronique

URL : http://journals.openedition.org/ress/367

DOI : $10.4000 /$ ress.367

ISSN : 1663-4446

\section{Éditeur}

Librairie Droz

Édition imprimée

Date de publication : 1 février 2005

Pagination : $35-46$

ISBN : 2-600-00958-2

ISSN : 0048-8046

Référence électronique

Henri Volken, « Mnémosyne érudite - la théorie comme mémoire », Revue européenne des sciences sociales [En ligne], XLIII-131 | 2005, mis en ligne le 05 novembre 2009, consulté le 01 mai 2019. URL : http://journals.openedition.org/ress/367 ; DOI : 10.4000/ress.367 


\section{Henri VOLKEN}

\section{MNÉMOSYNE ÉRUDITE LA THÉORIE COMME MÉMOIRE}

Le savoir n'est que mémoire.

Platon

Une approche naïve, mais très répandue, accorde à la mémoire la seule fonction de se souvenir. Mais la psycho-physiologie nous enseigne une mémoire distribuée, beaucoup plus complexe, avec des fonctions qui dépassent celles de la simple réactivation d'un souvenir, pour permettre représentation et modélisation. La mémoire nous donne les moyens d'établir notre identité, de projeter nos actes, d'échafauder des scénarios et surtout de comprendre et d'apprendre. Elle nous amène en permanence à reconsidérer des faits nouveaux à la lumière des faits anciens et réciproquement. Mais n'est-ce pas exactement la fonction de la théorie dans la démarche scientifique? Elle condense un savoir factuel en quelques principes ou lois, mais c'est elle aussi qui nous permet de construire des modèles de la réalité que nous étudions. Finalement c'est encore la théorie qui nous autorise à tirer des conséquences de ce que nous savons, de confronter celles-ci avec le monde extérieur et donc de stabiliser, mais aussi de faire évoluer notre savoir. La théorie fait de la science clairement plus qu'une simple accumulation d'informations.

Le texte qui suit est une invitation à se pencher sur cette analogie entre théorie et mémoire, en suivant notamment deux exemples historiques qui nous semblent bien illustrer cette parenté. Mais commençons par quelques questions élémentaires.

\section{IDENTITÉ DU SAVOIR}

Qu'est-ce que la sociologie? Qu'est-ce que la psychologie? Qu'est-ce que la biologie? Qu'est-ce que la physique? Ces questions si simples et si fondamentales donnent lieu bien sûr à des réponses très variées, selon les personnes interrogées. Mais d'une certaine façon, la difficulté de ces quatre questions peut être ressentie comme décroissante avec l'ordre dans lequel elles apparaissent ici. Il suffit pour s'en convaincre, de parcourir les définitions usuelles qu'en offrent les dictionnaires courants. Si la physique y est décrite comme la «science qui étudie les propriétés de la matière et établit des lois qui rendent compte des phénomènes naturels ${ }^{1}$, la biologie apparaît comme «science qui a pour objet l'étude des

\footnotetext{
Petit Robert, 1986
} 
phénomènes vitaux dans la cellule, dans l'individu et dans l'espèce $»^{2}$, faisant intervenir des notions moins simples en apparence. Dans la définition de la psychologie interviennent des concepts à première vue encore plus abstraits: la psychologie devient l'»étude scientifique des phénomènes de l'esprit, de la pensée, caractéristiques de certains êtres vivants (animaux supérieurs, homme) chez qui existe une connaissance de leur propre existence $»^{3}$. Quant à la sociologie, elle est l'»étude scientifique des faits sociaux humains, considérés comme appartenant à un ordre particulier et étudiés dans leur ensemble ou à un haut degré de généralité ${ }^{4}$. Dans cette dernière définition, d'apparence simple, surgissent pourtant des termes - «faits sociaux» notamment - qui font allusion à des propriétés de collectivités, donc d'entités plus abstraites encore que les individus dont s'occupe la psychologie.

Toute science est un savoir distribué sur un grand nombre de personnes et sur des supports matériels ou virtuels qui participent à sa mémoire. Ce savoir est constamment réorganisé, restructuré et diffusé à travers divers médias et institutions. On peut se demander comment une telle dispersion d'information permet encore de percevoir l'unité d'une science. Bien sûr les acteurs de cette science, et les éléments de la connaissance, sont hiérarchisés et on leur attribue une importance différenciée. L'organisation de la communication et de la diffusion structure également le corps d'une discipline. Mais la question fondamentale reste: Qu'estce qui fait l'identité d'un savoir? Est-ce l'accumulation des faits, observations et résultats? Et là, on se trouve confronté à la très ancienne difficulté de la sémantique de ces concepts. Un «fait physique» paraît plus simple à saisir qu'un «fait social» qui n'a pas la même permanence et la même saillance. Et de toute façon, problème ubiquiste, il n'y a pas de fait ni d'observation sans - au moins - un début de théorie. L'identité et la mémoire d'une science relèvent clairement d'une construction plus complexe qu'une simple accumulation. Néanmoins, il existe un aspect cumulatif évident dans chaque domaine de connaissance scientifique, qui progresse grâce au travail conjoint - globalement peu coordonné - de ses chercheurs. Il est vrai que d'autres formes de connaissance - religieuse par exemple n’ont pas cet aspect laborieux et procèdent directement par révélation ou illumination.

Nous avons évoqué, un peu arbitrairement, la physique, la biologie, la psychologie et la sociologie, les prenant comme exemples de domaines qui traitent d'objets et de phénomènes clairement distincts. Or il semble que nous ayons des appréciations très diverses de l'identité et de la constitution de ces savoirs. Les différences spécifiques de leurs définitions expliquent en partie cette disparité d'opinion. Jetons un bref coup d'œil sur l'image commune attribuée à chacune de ces sciences. La représentation de la physique comme une sorte de mosaïque à laquelle les chercheurs ajoutent patiemment de petites pièces sous forme de lois de plus en plus générales, est très répandue. Cette mosaïque a été soumise, il est vrai, à quelques violentes secousses et destructions dans son histoire, et son dessin a varié à maintes reprises, mais la reconstruction demeure à chaque fois de la

\section{Idem \\ Idem \\ ${ }^{4}$ Idem}


même nature, elle semble rester cumulative. Un exemple très simple le montre: un manuel de physique datant d'il y a un siècle, contient des informations dont la plupart doivent être apprises aujourd'hui encore par les étudiants du domaine. D'autres savoirs offrent un visage différent. La biologie se présente historiquement tout d'abord comme un grand amas d'observations concrètes, résultats d'expérimentations diverses, et de traces matérielles accumulées, comme des collections anatomiques ou zoologiques. C'est probablement l'apparition de la théorie de l'évolution qui lui a donné sa logique interne, et a donc contribué à lui donner le visage qu'elle possède aujourd'hui. La psychologie nous éloigne encore des objets matériels et visibles. Ses propres objets sont de nature plus complexe puisqu'il s'agit de phénomènes de la pensée et de comportements d'êtres humains. Le savoir dépend à première vue davantage des courants dominants qui imposeraient leurs concepts, leurs méthodes et leur champ expérimental. Finalement, la tâche de considérer le savoir sociologique comme collection d'observations objectives apparaît encore plus difficile. Car ici non seulement il est question d'observer des comportements, mais encore s'agit-il de comportements non pas d'individus, mais de groupes sociaux, qui sont des agents virtuels et donc pas directement observables. L'importance dans la constitution du savoir sociologique des écrits de ses chercheurs, de leur notoriété changeante et des intérêts subjectifs des auteurs, donne, de l'extérieur du moins, à la sociologie une apparence de palimpseste plutôt que de mosaïque.

L'idée d'un manuel ancien qui contiendrait des informations toujours actuelles, ne paraît pas imaginable, en tout cas clairement dans les deux derniers cas, qu'il s'agisse de psychologie ou se sociologie. Cette remarque ne s'applique qu'à la notion de manuel bien sûr et ne concerne nullement l'intérêt et l'importance des écrits plus anciens dans ces différentes sciences. Le contraste entre ces sciences, même en ce qui concerne la physique, avec les mathématiques est saisissant. Il suffit de penser à certains résultats attribués à Thalès ou Pythagore - donc datant de plus de deux millénaires - et de constater leur actualité. Mais surtout, l'enseignement de la géométrie est encore basé essentiellement sur les travaux d'Euclide et sur la forme axiomatique de son enseignement. Cette forme axiomatique a influencé durablement la pensée mathématique et dans une large mesure, la pensée scientifique. Elle constitue surtout l'un des exemples paradigmatiques de mémorisation d'un savoir et d'une pratique scientifique. En ce sens elle joue un rôle exemplaire dans la suite de notre discussion.

Comment un domaine de la connaissance constitue-t-il son identité? Nous allons voir, et c'est la principale thèse de ce texte, que la théorie représente une forme efficace de mémoire, indispensable dans l'organisation et la transmission du savoir. Pour illustrer ce point de vue, voici, en grandes lignes, l'exemple des tribulations de notre connaissance astronomique du système solaire. Cet exemple est particulièrement important par la nature à la fois symbolique et scientifique de ce savoir, qui est à la base de toutes nos cosmologies et d'une certaine partie de nos mythes.

\section{LE SYSTÈME SOLAIRE}

L'observation des astres a toujours été une source d'émerveillement autant que de questionnement. C'est probablement l'aspect de périodicité qui a intrigué le 
plus profondément. Certains événements célestes se répètent apparemment à intervalles réguliers, ce qui a pu suggérer - à celui qui voulait comprendre le comportement des astres - la comparaison avec une horloge de taille cosmique. Mais les intervalles observés peuvent être très différents, il suffit de penser à l'alternance du jour et de la nuit, des phases lunaires ou du passage des saisons. Et les longueurs des intervalles correspondant à différents phénomènes, ne sont souvent pas des multiples (simples) les uns des autres, ce qui rend difficile la compréhension du système. D'autant plus que certains phénomènes ne sont pas, ou ne semblent pas, périodiques ni même réguliers.

Deux voies ont été explorées pour comprendre le mécanisme du système solaire. La simple observation directe, qui a débouché sur l'attitude expérimentaliste qui consiste à collectionner les traces des observations, et l'introspection, qui pousse à rechercher une explication exclusivement à l'intérieur de son propre système de pensée. Des tablettes d'argile trouvées en Mésopotamie et datant de plus de deux millénaires avant notre ère, attestent d'une première forme de mémorisation de l'observation systématique des phénomènes célestes. Toute la science était contenue dans ces traces gravées qui permettaient - par interpolation et extrapolation - de calculer des calendriers ou prévoir des événements réguliers.

Parallèlement à cette collecte de données empiriques, une autre approche est apparue. Les philosophes grecs, à commencer par Thalès, ont cherché, de leur côté, une explication du système solaire - et du monde - dans des abstractions très générales, qui n'avaient pas pour origine l'observation empirique. Ils visaient des principes fondamentaux, à partir desquels une explication pour un phénomène spécifique pouvait être tirée par le raisonnement. Pour Thalès, par exemple, à l'origine de toute chose il y avait l'eau, pour Anaximandre, c'était l'infini, un principe complexe et intriguant. Les premiers modèles «scientifiques» du mouvement des planètes, d'inspiration géométrique, étaient l'œuvre d'Eudoxe, et de Ptolémée, tous deux de brillants mathématiciens.

La connaissance du système solaire a réellement pris son envol au tournant du $\mathrm{XVII}^{\mathrm{e}}$ siècle, par la combinaison de ces deux approches. La richesse et la précision des observations a sous-tendu le développement de théories qui permettaient d'expliquer et d'extrapoler tout en constituant par la suite une fabuleuse mémoire qui intégrait toutes les traces empiriques. L'évocation de quelques grands noms va nous servir à décrire l'essentiel de la situation.

Galilée, dont les préoccupations étaient pourtant avant tout terrestres, a contribué à la connaissance de la dynamique des astres, en mettant en doute deux principes largement acceptés avant lui et qui concernaient le mouvement. Il s'agit d'abord de l'idée d'un «impetus» que posséderait un corps en mouvement et qui se perdrait peu à peu. Le deuxième principe couramment admis stipulait que les corps lourds tombaient plus vite que les corps légers. Galilée remplace ces principes par deux lois qui ont une importance fondamentale pour la suite: la loi de l'inertie et la loi de la chute libre, qui allait marquer l'entrée du temps comme variable explicite dans la physique. Ce qui est radicalement nouveau dans la démarche de Galilée, c'est la place que prend l'expérimentation comme critère de vérification de ses théories. Celles-ci se construisent sur la base de ses observations expérimentales et les résument. Cette construction se fait par des mécanismes d'induction en se basant largement sur les possibilités du langage mathématique utilisé pour la représentation des concepts impliqués. C'est princi- 
palement la notion de fonction qui permet de mémoriser les points correspondant aux observations par un objet synthétique unique. Une fonction codifie un comportement - l'orbite d'une planète par exemple - par son expression mathématique, qui formalise ainsi la loi sous-jacente. Une formule devient un savoir condensé, le lieu de la mémoire de tout un ensemble de connaissances empiriques accumulées.

Dans la pensée astronomique de Galilée, l'un des points importants est dû à l'un de ses précurseurs, l'astronome polonais Copernic, qui affirma le caractère héliocentrique du système solaire dans son ouvrage De Revolutionibus Orbium Coelestiom (1543). Cette idée avait déjà surgi dans l'antiquité, mais avait été occultée par le modèle du monde de Ptolémée. Copernic replaça le soleil au centre du système, déclara que les planètes se déplaçaient sur des orbites circulaires dans des plans légèrement différents les uns par rapport aux autres. Cette description qualitative, incomplète et partiellement fautive avait cependant le mérite de replacer la discussion cosmologique dans des voies correctes. Aux spéculations de Copernic allait répondre la méticulosité empirique de Tycho Brahé, qui est à l'origine d'une collection essentielle de données précises sur la position des astres.

Comme Galilée, Brahé, astronome danois, croyait à l'observation de la réalité plutôt qu'aux spéculations abstraites pour établir la connaissance de la nature. Sur l'île danoise de Hven, il consacra une vingtaine d'années à observer minutieusement les planètes et les étoiles avec des instruments qu'il s'était construit luimême. La science astronomique de Brahé se résumait, après cela, à une impressionnante collection de mesures ${ }^{5}$ accumulées. Celle-ci constituait à la fois sa mémoire et son identité. Sous cette forme, l'astronomie permettait d'expliquer et de prévoir des phénomènes célestes par des manipulations laborieuses de quantités de données. De part son support matériel, elle était fragile et sa diffusion restait très limitée. Nous allons voir comment la théorisation, et plus tard la mathématisation, ont permis de transformer et résumer ce savoir en une mémoire à long terme, pour emprunter un concept de biologie.

Après des controverses avec le roi du Danemark, Tycho Brahé s'installe à Prague où il a pour collègue le jeune Kepler. Ce dernier profite de la richesse et la précision des observations de Brahé pour en extraire les formes mathématiques capables de les résumer. Il adopte le modèle héliocentrique de Copernic et s'apprête à décrire les données relatives à l'orbite de Mars par une trajectoire circulaire lorsqu'il constate une déviation des positions mesurées par Brahé par rapport à sa trajectoire de plus de 8 minutes d'arc, dépassant de loin la marge d'erreur des mesures utilisées. Il fut obligé ${ }^{6}$, après ce constat, de modifier les trajectoires de son modèle en introduisant une certaine excentricité, passant ainsi des orbites circulaires à des orbites elliptiques, dont l'un des foyers était occupé par le soleil. A l'aide de ces nouveaux objets mathématiques, il réussit à résumer l'entier des mesures empiriques dont il disposait. Mieux, il pouvait calculer les positions des planètes pour n'importe quel moment de l'année, complétant par là les observations de Tycho Brahé. Il avait consolidé l'information contenue dans la mémoire

Tycho Brahé avait obtenu ses mesures avec une précision d'une minute d'arc.

' Il écrivit à ce propos: «Sur ces 8 minutes je construirai un monde». 
fragile des données empiriques en une mémoire persistante beaucoup plus flexible, complète et opérationnelle, une théorie exprimée dans un langage mathématique. Tout le savoir de Tycho Brahé et toutes les observations astronomiques accumulées jusque là, se trouvent ainsi résumés dans les trois lois du mouvement planétaire de Kepler, découvertes entre 1601 et 1618 et publiées dans Harmonicus Mundi (1619):

L'orbite d'une planète relative au soleil se trouve dans un plan fixe contenant le soleil.Chaque planète se déplace autour du soleil en suivant une ellipse dont le soleil se trouve à l'un des foyers.

Le vecteur reliant le soleil à la planète balaie des aires égales pour des intervalles de temps égaux.

Le carré de la période de révolution d'une planète est proportionnel à la troisième puissance du plus grand des demi-axes de l'ellipse.

Or si Kepler était à l'origine de la première description cinétique du système solaire, son modèle n'incluait pas une explication de la cause du mouvement observé et n'était pas exempt d'un certain mysticisme. Il faut attendre Newton et son œuvre, Philosophiae naturalis principia mathematica (1687), pour faire une synthèse de tout le savoir astronomique établi avant lui, avec quelques intuitions fondamentales permettant de relier la notion de mouvement avec celle de force. Dans sa fameuse loi sur l'accélération, Newton explicite sa vision - dynamique du lien qui existe entre les deux concepts:

«La masse d'un corps, multipliée par l'accélération est égale à la résultante des forces agissant sur lui »ou, en termes mathématiques, par l'équation différentielle: $\mathbf{F}=\mathbf{m a}$

A l'aide de cette découverte, Newton interpréta les lois de Kepler de manière astucieuse et originale pour aboutir finalement à sa théorie générale de la gravitation. Pour cela il utilisa un théorème essentiel qui montre que la force de gravitation d'un corps de symétrie sphérique reste la même si toute la masse est concentrée en son centre. Cela permet de s'abstraire de la réalité matérielle des objets célestes et de se rapprocher de la fiction mathématique du point masse. Les objets réels sont représentés par des concepts mathématiques et par conséquent ils bénéficient du traitement opérationnel d'un calcul efficace. En ayant identifié les forces qui provoquent la chute des objets sur terre avec celles qui provoquent les mouvements des planètes, Newton franchit un pas fondamental dans notre compréhension du monde car il réussit par-là à simplifier davantage encore sa théorie, réduisant une nouvelle fois la quantité d'information constitutive de la science astronomique. Cette réduction s'est faite en partie grâce à l'aide du puissant formalisme mathématique du calcul différentiel, que Newton a inventé (en parallèle avec Leibniz, mais indépendamment de lui) à cette occasion.

Non seulement la théorie générale de la gravitation et son application à la dynamique du système solaire permettait aux scientifiques d'oublier les détails des observations de Brahé, mais elle ouvrait la voie aux vérifications expérimentales. Newton par exemple a utilisé le mouvement de la lune autour de la terre pour vérifier ses lois. Pour cela il lui suffisait d'appliquer son modèle aux données astronomiques connues et de calculer des phénomènes observables. On voit 
apparaître dans cet exemple clairement la double fonction de la théorie, d'une part comme mémoire permettant de stocker une grande quantité d'information en la condensant par le moyen de lois universelles, puis de formules mathématiques, et d'autre part comme simulateur opérationnel de la réalité observée.

Newton a introduit sa mécanique céleste sous forme axiomatique, ce qui est le mode paradigmatique de la fonction mnésique d'une théorie formalisée. Son système contient tout le savoir factuel et théorique concernant la mécanique céleste et la gravitation, auquel s'ajoutent certains postulats de nature plus épistémologiques et dictés par un besoin de cohérence formelle. Sans eux la théorie n'a pas de véritables fondements solides. Il s'agit des postulats du temps absolu et de l'espace absolu, remis en question plus tard par l'approche relativiste de la mécanique. Ces deux axiomes sont nés de considérations logiques concernant la théorie et ne constituent pas une mémoire pour les traces d'observations et d'expériences passées mais soutiennent l'ensemble de l'édifice conceptuel.

\section{L'AXIOMATIQUE}

La démarche axiomatique semble être née en Grèce aux $\mathrm{IV}^{\mathrm{e}}$ et $\mathrm{III}^{\mathrm{e}}$ siècles avant notre ère avec les travaux d'Euclide et de ses successeurs et reste aujourd'hui encore une forme privilégiée de transmission du savoir mathématique. Bien que la géométrie d'Euclide s'appuie sur des travaux plus anciens, notamment ceux de Thalès, de Pythagore et d'Eudoxe, la forme particulière dans laquelle elle se présente aura une influence durable sur l'écriture et la pensée mathématique. Il s'agit historiquement d'une des premières tentatives systématiques de condensation d'un savoir en quelques principes fondamentaux - appelés aujourd'hui axiomes, mais qu'Euclide appelait postulats - à partir desquels on peut déduire tous les éléments de ce savoir. Cette manière de mémorisation offre des perspectives fascinantes et pose des problèmes qui animent aujourd'hui encore les discussions épistémologiques, en particulier à propos des fondements des mathématiques. Elle est à l'origine de la méthode hypothético-déductive qui soustend une partie de la démarche scientifique depuis quelques siècles.

On peut caractériser en grandes lignes un système axiomatique par l'équation:

$$
\text { Axiomes }+ \text { Logique }=\text { Théorèmes }
$$

Cette structure implique une sélection d'axiomes, de postulats ou de notions communes dans le langage d'Euclide, qui contient en germe tout le savoir d'une discipline, ainsi qu'un système déductif qui permet d'accéder à des connaissances dérivées. Chacun de ces deux concepts soulève un certain nombre de problèmes. Tout d'abord le statut et le choix des axiomes. Si pour Euclide un postulat était indémontrable, cela signifiait simplement que dans sa vision syntaxique de la notion de conséquence logique, il n'y avait aucune nécessité de le démontrer. Il est probable qu'Euclide attribuait à ces objets une certaine forme d'évidence que les théorèmes, les propositions dérivées, ne possédaient pas. Plus tard, dans une approche sémantique, les axiomes représentaient des vérités qui s'imposeraient à nous par une sorte d'évidence empirique. Depuis le XIX ${ }^{\mathrm{e}}$ siècle, leur statut sera plus libre et arbitraire comme nous allons le voir. 
Le problème du choix des axiomes est crucial dans notre analogie avec la mémoire d'une science. Car ce choix représente le véritable mécanisme de mémorisation. Pour Euclide, il s'agissait de ne retenir du savoir géométrique étendu de son époque que quelques principes fondamentaux, suffisants pour reconstruire au moins ce savoir. Cette sélection constitue un mécanisme d'encodage, analogue aux logiciels de compactification de données que nous utilisons sur nos ordinateurs et qui réduisent radicalement la place de mémoire occupée par des fichiers son ou image. On ignore comment Euclide a procédé, mais il est probable que c'est par une analyse extrêmement précise des démonstrations existantes et de celles qu'il avait découvertes, qu'il a finalement arrêté son choix sur les postulats qui figurent dans ses Eléments. Cette réduction des connaissances à une liste relativement courte de notions communes et de postulats, constitue une véritable prouesse intellectuelle. Il suffit de rappeler pour s'en convaincre que pendant plus de deux millénaires le savoir géométrique a été transmis essentiellement sous cette forme.

Mais rien ne sert de rendre compact le savoir - pour le stocker en mémoire si nous n'avons pas les moyens d'y avoir accès par la suite. Ce mécanisme de récupération est donné dans le contexte de l'axiomatique par un système déductif, c'est-à-dire une logique. Pendant longtemps ce système était constitué des éléments de la logique grecque, essentiellement de la logique d'Aristote et des fragments de la logique des connecteurs développée par les Stoïciens. Bien plus tard, avec la formalisation de la logique au cours des $\mathrm{XIX}^{\mathrm{e}}$ et $\mathrm{XX}^{\mathrm{e}}$ siècles, grâce aux travaux de Boole, Frege, Peano, Russel et de bien d'autres, c'est une version plus mathématisée de la logique qui s'est imposée. Ce moteur déductif ne permet pas seulement de retrouver les théorèmes connus et mémorisés, mais donne la possibilité d'en trouver de nouveaux, tout en fournissant une version codifiée de la notion de preuve. Le système axiomatique constitue donc une mémoire très condensée et opérationnelle pour un ensemble de connaissances, à condition que celles-ci puissent être exprimées en un langage univoque et si possible formalisé.

Dans un système axiomatique, le concept de preuve tient lieu d'idée de vérité. L'existence de la preuve d'un théorème ne signifie rien d'autre, que le fait que celui-ci a pu être dérivé selon les critères admis par les règles de déduction. Un théorème a donc pu être récupéré dans la mémoire à l'aide de ces règles ou alors il constitue une nouveauté qui accroît le domaine du savoir. L'exigence minimale dans ce cas est la consistance, le fait qu'on ne puisse pas dériver une proposition et sa négation. Il s'agit de l'une des contraintes élémentaires imposées à un système axiomatique classique. Mais comment établir la consistance d'un système? Bien sûr, si dans le cours d'une dérivation il apparaît un théorème aussi bien que sa négation, alors l'inconsistance est avérée. Dans le cas contraire, si aucune contradiction n'est apparue après un nombre fini de théorèmes déduits, la consistance n'est pas encore garantie. D'où l'introduction d'un critère de consistance basé sur une notion sémantique de modèle. L'introduction d'un modèle se fait par une interprétation des termes indéfinis de la théorie qui convertit les axiomes en propositions vraies. Dans le cas où le système axiomatique provient d'un domaine concret, le modèle sera précisément ce domaine et la vérité sera établie par une simple vérification. Pour l'axiomatisation d'un domaine abstrait, l'établissement de la consistance est plus problématique puisque le monde 
«concret» du modèle, reste lui-même abstrait. On pourra au mieux établir dans ce cas une forme de consistance relative.

Lorsqu'un domaine de connaissance est «mémorisé » par une théorie, supposons ici par une théorie axiomatique, l'une des questions importantes est de savoir si l'on peut retrouver tous les éléments de ce savoir. Ce problème est central dans le développement et l'utilisation des approches axiomatiques. Il s'agit de la question de la complétude. On a pu montrer par exemple que le système original des Eléments d'Euclide n'était pas complet. Ce n'est qu'au début du $\mathrm{XX}^{\mathrm{e}}$ siècle que Hilbert a proposé un système revu et corrigé d'axiomes pour la géométrie. La question est importante dans la perspective adoptée ici, qui consiste à mettre en avant l'analogie théorie/mémoire. Gödel a montré en 1931 dans son célèbre théorème de l'incomplétude que toute théorie possédant un certain nombre de propriétés formelles ${ }^{7}$, ne peut pas être complète. C'est-à-dire qu'elle ne permet pas de retrouver, par déduction, tous les éléments du domaine dont elle constitue la mémoire. Cependant cette restriction ne s'applique qu'aux systèmes possédant un certain développement mathématique.

Parmi les axiomes choisis pour fonder un système axiomatique, il se peut que certains soient inutiles dans le sens où ils seraient déjà des conséquences d'autres axiomes. C'est la question de l'indépendance des axiomes qui a secoué le monde mathématique du XIX ${ }^{\mathrm{e}}$ siècle. Il se trouve que le cinquième postulat d'Euclide semble posséder un statut ambigu. Ce postulat stipule que par un point extérieur à une droite on peut faire passer une et une seule droite parallèle à la première. Or Euclide lui-même pensait que ce postulat différait des autres par sa nature. Comme il ne lui paraissait pas suffisamment «évident» et n'osant pas le déclarer comme une simple hypothèse, Euclide a vainement essayé de le considérer comme théorème et de le démontrer à partir des autres axiomes. La question est réapparue en force dans le discours mathématique lorsque Gauss, Lobatchevski, Bolyai et Riemann on tenté de clarifier le statut de ce postulat au XIX $\mathrm{X}^{\mathrm{e}}$ siècle. Après d'infructueuses tentatives de démontrer à son tour le cinquième postulat à partir des autres, Lobatchevski remplaça ce postulat par le suivant: par un point extérieur à une droite on peut faire passer une infinité de droites parallèles à la première. Il réussit à trouver une interprétation de son nouveau système dans un modèle géométrique existant, montrant par-là la consistance - relative - de sa nouvelle géométrie non euclidienne. Le postulat des parallèles s'était donc révélé indépendant des autres postulats. Il était établi qu'il mémorise bien une propriété qui n'est pas contenue dans les autres axiomes.

\section{THÉORIE ET MÉMOIRE}

A l'aide de l'illustration fournie par ces deux exemples, il a été possible d'esquisser ce qu'on pourrait appeler la fonction mnésique de la théorie. En voici le rappel. Si l'on admet que tout savoir se constitue à partir de l'observation de régularités, de structures remarquables, de coïncidences ou autres faits, le pas suivant dans la démarche scientifique ne peut être que la catégorisation. Il s'agit à la fois

Cf [1] 
d'une création de concepts et d'une accumulation et réorganisation des observations à la lumière de ce cadre nouveau. Pratiquement à l'issue de cette phase, la mémoire est constituée de recueils d'observations, de rapports d'expériences, de comptes rendus de recherches, de tableaux statistiques, dont les traces se trouvent dans des publications ad hoc ou des revues, ou qui sont véhiculés par des médias rapides comme internet, accompagnés de généralisations, d'inductions et d'hypothèses. La durée de vie de ces informations peut être relativement courte.

Historiquement, cette première étape ne fut pas toujours suivie d'une phase de théorisation dans le sens d'une science déductive. Le mouvement initié par Francis Bacon au début du XVII ${ }^{\circ}$ siècle tendait à une accumulation systématique de faits et d'observations afin d'augmenter les connaissances scientifiques et techniques $^{8}$. Cet empirisme rationnel, planifié, visait moins le savoir abstrait que la maîtrise pratique de la nature. Mais surtout la systématisation rigoureuse de l'expérimentation devait produire une base inductive pour l'argumentation. Bacon s'opposait fortement à une vision déductive de la science'. Or cette méthodologie n'a produit en fin de compte qu'une accumulation de connaissances sous forme de tables, d'inventaires et de répertoires, dont la quantité n'en favorisait pas l'emploi comme outil d'argumentation, en dehors d'évidentes tentatives de généralisation. On peut lui accorder le crédit d'avoir créé une classification systématique des domaines du savoir, ouvrant par-là quelques pistes nouvelles de recherche. Mais l'absence de structure théorique déductive, capable de mémoriser l'ensemble des données empiriques et de rendre possible une confrontation de ces données avec des conséquences de la théorie, rend l'entreprise de Bacon inefficace.

Nous en venons donc à la place que tient la théorisation dans la démarche scientifique dans une étape ultérieure. Après la phase de collecte de résultats et d'observations, et d'une mémorisation temporaire de ces données, ainsi qu'une phase de catégorisation, commence l'ébauche d'une véritable théorie. Celle-ci crée un réseau à partir des concepts définis par la classification avec des liens déductifs et la possibilité de définir de nouvelles notions à partir de ces concepts. Ce schéma se retrouve dans l'exemple du système solaire que nous avons évoqué. Les observations méticuleuses des positions des planètes effectuées par Brahé, transformées par catégorisation en orbites, ont ensuite permis de leur associer une forme théorique - en l'occurrence l'ellipse - exprimable en termes mathématiques. L'équation de l'ellipse représentant l'orbite, non seulement résume les mesures effectuées, mais offre un modèle calculatoire qui peut servir à l'extrapolation et la prédiction. La comparaison des ellipses correspondant à chaque planète et des plans dans lesquels elles évoluent, procure un modèle mathématique plus complet du système solaire. Nous voyons clairement la fonction que joue ici la théorie: non seulement elle résume et codifie les observations passées, mais elle est un instrument de représentation, de modélisation, qui autorise l'extrapolation et aussi la déduction de conséquences logiques. Celles-ci à leur tour permettent, par une confrontation avec la nature, la vérification ou la falsification, et finalement rendent possible l'évolution de la théorie elle-même.

\footnotetext{
Il faut rapprocher d'une certaine façon la démarche de Tycho Brahé de ce courant.

Cf [2]
} 
L'axiomatique, l'autre exemple que nous avons présenté, ou plutôt l'autre classe d'exemples, illustre des aspects différents de la fonction de mémoire propre à la théorie. En particulier, nous avons vu que les axiomes, éléments fondateurs du savoir, ne sont pas univoquement déterminés et peuvent être choisis librement dans un certain domaine, en tenant compte des contraintes logiques: le système résultant doit être consistant, et si possible complet. Il y a donc, en tout cas dans l'approche axiomatique, de nombreuses façons de codifier un domaine de connaissances. Le choix des éléments générateurs qui sous-tendent la mémoire n'est pas univoque.

Le rôle de la théorie dans la double activité de mémorisation et la constitution de l'identité d'une science est itératif. Nous avons parlé jusqu'ici un peu naïvement d'une phase d'observation, comme si celle-ci pouvait se passer entièrement de théorie. Mais c'est au contraire parce que la théorie a permis d'intérioriser des faits et des hypothèses que les nouvelles observations sont pertinentes. La théorie guide les observations et celles-ci permettent à la théorie de constituer l'identité de la science concernée. La théorie en est conjointement la mémoire et la structure organisationnelle. Une simple juxtaposition de faits ne saurait en aucun cas constituer une science, c'est-à-dire un domaine évolutif du savoir où l'argumentation rationnelle a sa place et où une confrontation avec la partie du monde concernée est possible. Vu sous cet angle, toute science est cumulative à travers la mémoire constituée par sa théorie. Si parfois elle ne semble pas l'être, c'est que l'évolution de la théorie peut avoir une dynamique d'apparence chaotique, avec des phases de consolidation suivies de phases de bifurcation. La théorie comme mémoire doit être parfois capable d'oubli.

Si une équation peut résumer d'innombrables données numériques comme l'a montré Kepler, cela démontre l'efficacité de la formalisation dans l'activité mnésique, et notamment de la formalisation en termes mathématiques. La mathématisation d'une théorie, lorsqu'elle est possible, rend le savoir compact et opérationnel. Les mathématiques, producteurs de formes, offrent à la science son capacité de mémorisation et en même temps au chercheur une extension de ses aptitudes de représentation et de ses facultés d'imagination. La mathématisation de leur démarche explique dans une mesure importante le succès de l'astronomie et de la physique en général. Mais qu'en est-il des sciences humaines?

Nous avons brièvement évoqué plus haut les difficultés inhérentes aux sciences sociales, qui rendent celles-ci rétives à la formalisation: notamment la non-linéarité de leurs interactions et la nature de leurs objets, qui sont souvent des collectivités, donc des concepts d'un ordre de complexité supérieur, et par conséquent impossibles à observer directement. N'étant pas directement observables mais devant être reconstruits, le comportement qu'on pourrait attribuer à ces objets peut être perçu et décrit par chaque chercheur différemment. Ce qui signifie que les informations accumulées dépendent du point de vue adopté, et ne sont utilisables véritablement que par ceux qui partagent ce point de vue. La mémorisation s'en trouve distribuée sur des théories concurrentes. A cela s'ajoute la diversité des concepts utilisés et l'ambiguïté de leur interprétation sémantique. Néanmoins, à voir les formidables richesses accumulées depuis un ou deux siècles par ces sciences relativement jeunes, on peut s'attendre à voir émerger des théories permettant d'unifier et de préciser ces domaines, donc de structurer leur mémoire. L'une des voies qui semblent indiquer une tendance dans ce sens est 
l'apparition des sciences de la complexité ${ }^{10}$. Dans ce cadre, des notions spécifiques sont apparues - émergence, adaptation, non-linéarité, intelligence distribuée, réseau en sont quelques exemples - qui pourraient capter certaines de ces réalités qui rendent les sciences sociales difficiles à intégrer dans un cadre conceptuel unique. Peut-être verra-t-on un jour le discours de Durkheim sur la solidarité dans la société, traduit - du moins dans sa dimension fonctionnelle - en termes d'effets non linéaires et collectifs d'un système complexe ? ${ }^{11}$

Université de Lausanne

\section{RÉFÉRENCES}

[1] H.D. EBBINGHAUS, J. FLUM \& W. THOMAS, Mathematical Logic, Springer, 1984.

[2] Thomas S. KUHN, The essential tension, University of Chicago Press, 1977.

[3] K. MAINZER, Thinking in complexity, 4th edition, Springer, 2004.

[4] H. A. SIMON, Les sciences de l'artificiel, Gallimard 2004.

${ }_{11} \mathrm{Cf}[3,4]$

${ }^{11}$ Cf [3] 